Supplement of Hydrol. Earth Syst. Sci., 22, 5227-5241, 2018

https://doi.org/10.5194/hess-22-5227-2018-supplement

(C) Author(s) 2018. This work is distributed under

the Creative Commons Attribution 4.0 License.

(c) (1)

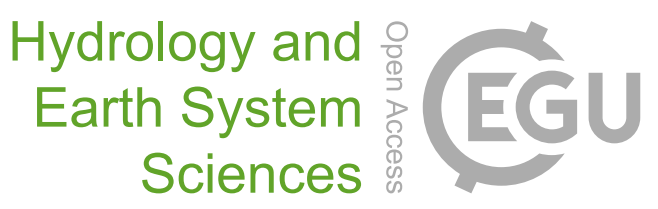

Supplement of

\title{
Land cover effects on hydrologic services under a precipitation gradient
}

\author{
Ane Zabaleta et al. \\ Correspondence to: Ane Zabaleta (ane.zabaleta@ehu.eus)
}

The copyright of individual parts of the supplement might differ from the CC BY 4.0 License. 


\section{Supplementary material}

Figure S1: Hydrographs for the 20 catchments in Table 1 for the hydrological year 2000-2001. The meaning of some of the calculated hydrological indicators is also indicated in the figure.

Figure S2: Boxplots representing the statistics of a) annual and b) seasonal precipitation for the 20 studied catchments during the hydrological years considered. $\mathrm{A}=$ Autumn, $\mathrm{W}=$ Winter, $\mathrm{Sp}=$ Spring and $\mathrm{Sm}=$ Summer.

Figure S3: Linear regressions obtained between a) annual precipitation ( $\mathrm{YP}, \mathrm{mm})$ and runoff $(\mathrm{YR}, \mathrm{mm}) \mathrm{b}$ ) precipitation from spring and winter $(\mathrm{SpP}+\mathrm{WP})$ and average discharge in spring $(\mathrm{Sp} 50 \mathrm{~m}) \mathrm{c}$ ) precipitation from winter and autumn (WP + AP) and wintertime high flows (W90m) and d) precipitation from spring and winter (SpP + WP) and low flows in spring $(\mathrm{Sp} 10 \mathrm{~m})$. 


\section{Figure S1}

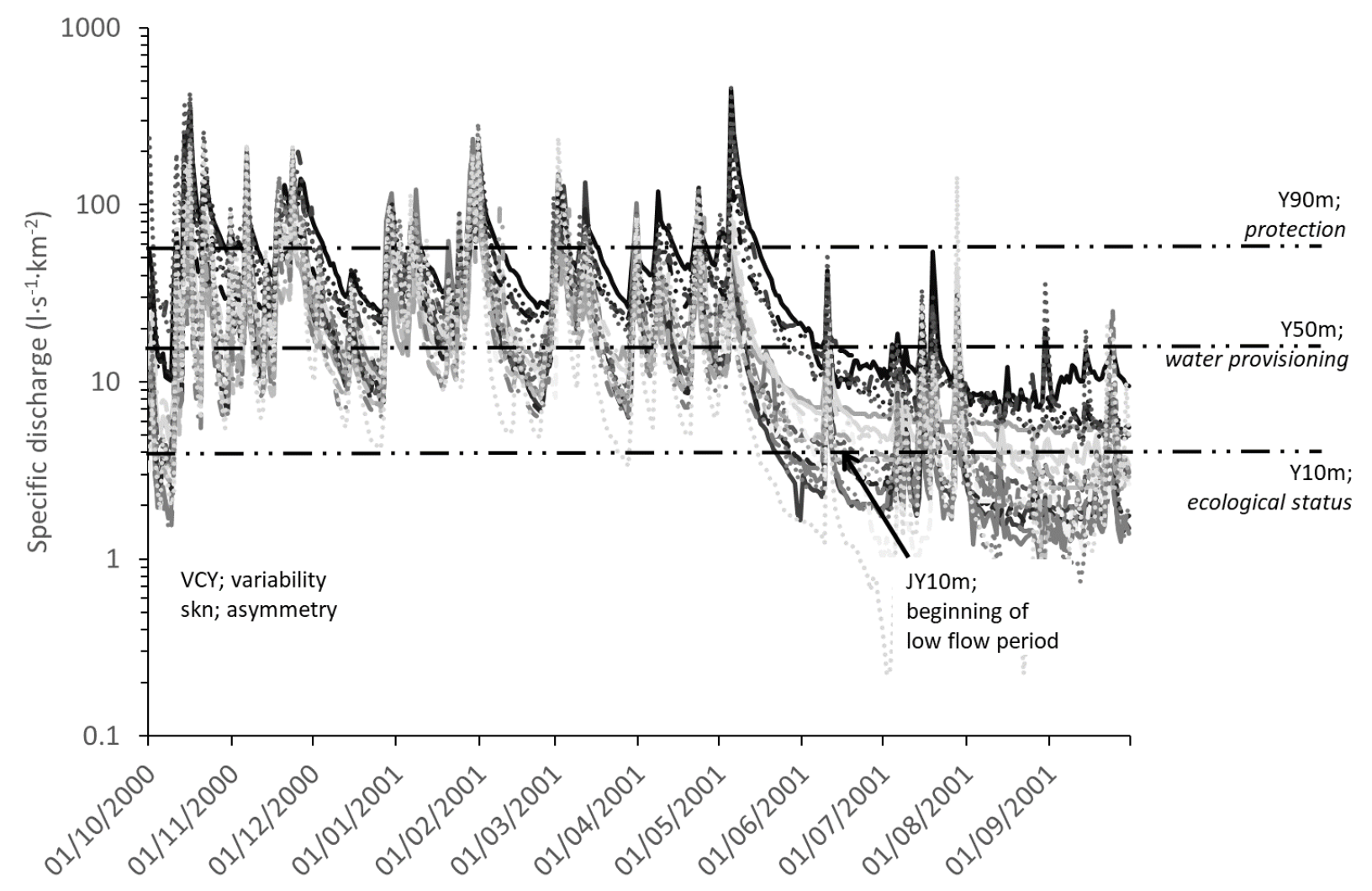

Figure S1: Hydrographs for the 20 catchments in Table 1 for the hydrological year 2000-2001. The meaning of some of the calculated hydrological indicators is also indicated in the figure. 
Figure S2
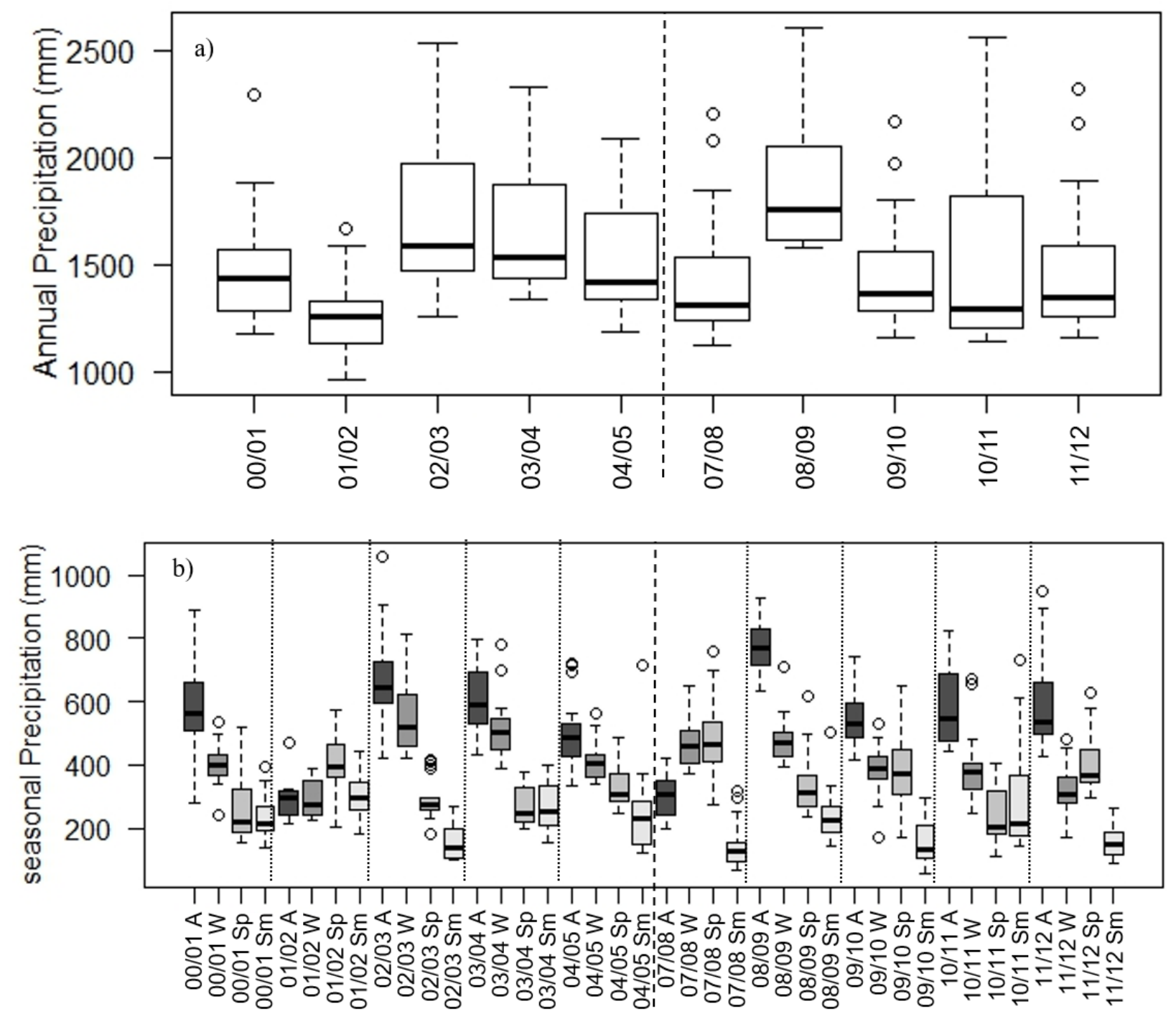

Figure S2: Boxplots representing the statistics of a) annual and b) seasonal precipitation for the 20 studied catchments during the hydrological years considered. $\mathrm{A}=$ Autumn, $\mathrm{W}=\mathrm{Winter}, \mathrm{Sp}=$ Spring and $\mathrm{Sm}=$ Summer. 
Figure S3
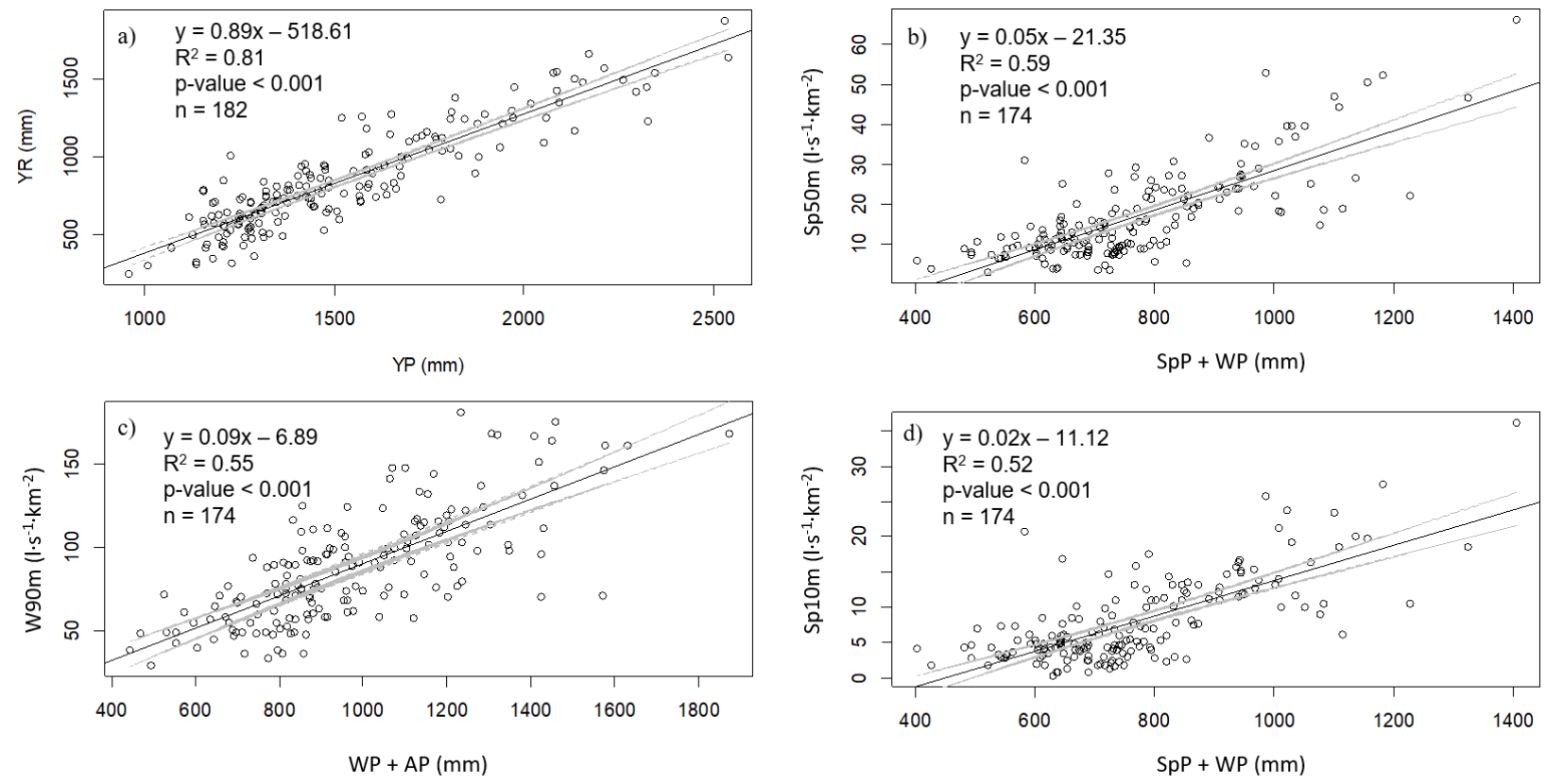

Figure S3: Linear regressions obtained between a) annual precipitation ( $\mathrm{YP}, \mathrm{mm}$ ) and runoff $(\mathrm{YR}, \mathrm{mm}) \mathrm{b}$ ) precipitation from spring and winter $(\mathrm{SpP}+\mathrm{WP})$ and average discharge in spring $(\mathrm{Sp} 50 \mathrm{~m}) \mathrm{c}$ ) precipitation from winter and autumn (WP + AP) and wintertime high flows (W90m) and d) precipitation from spring and winter (SpP + WP) and low flows in spring $(\mathrm{Sp} 10 \mathrm{~m})$. 
Table S1: Multiple regression models.

a) Multiple regression model for annual average flows (Y50m) considering alternative land cover and its interaction with annual precipitation (YP).

\begin{tabular}{lrrrrc}
\hline & Estimate & Std. Error & $\mathrm{t}$ value & $\mathrm{p}$ value & Significance \\
\hline (Intercept) & 50.67649 & 21.3388 & 2.3749 & 0.01862 & $*$ \\
YP & -0.02049 & 0.0150 & -1.3635 & 0.17446 & \\
Native & -0.80268 & 0.2677 & -2.9985 & 0.00310 & $* *$ \\
Exotic & -0.56718 & 0.2329 & -2.4354 & 0.01586 & $*$ \\
Pasturelands & -0.81356 & 0.2181 & -3.7296 & 0.00026 & $* * *$ \\
$\mathrm{I}($ Native * YP) & 0.00046 & 0.0002 & 2.6403 & 0.00902 & $* *$ \\
$\mathrm{I}($ Exotic *YP) & 0.00030 & 0.0002 & 1.8558 & 0.06513 & $*$ \\
$\mathrm{I}($ Pasturelands * YP) & 0.00057 & 0.0002 & 3.4285 & 0.00075 & $* * *$ \\
\hline
\end{tabular}

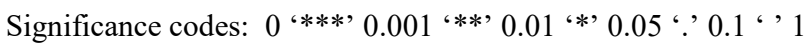

Residual standard error: 3.96 on 178 degrees of freedom

Multiple R-squared: 0.7791， Adjusted R-squared: 0.7704

F-statistic: 89.69 on 7 and 178 DF, p-value: <2.2e-16

b) Multiple regression model for average discharge for spring $(\mathrm{Sp} 50 \mathrm{~m})$ considering alternative land cover and its interaction with seasonal precipitation (spring + winter precipitation, SpPt).

\begin{tabular}{lrrrrc}
\hline & Estimate & Std. Error & $\mathrm{t}$ value & $\mathrm{p}$ value & Significance \\
\hline (Intercept) & 84.01452 & 43.1914 & 1.9452 & 0.05345 & $\cdot$ \\
SpPt (SpP+WP) & -0.06791 & 0.0586 & -1.1585 & 0.24833 & \\
Native & -1.44268 & 0.5367 & -2.6879 & 0.00792 & $* *$ \\
Exotic & -0.86076 & 0.5230 & -1.6457 & 0.10172 & \\
Pasturelands & -0.98659 & 0.3952 & -2.4965 & 0.01352 & $*$ \\
$\mathrm{I}($ Native * SpPt) & 0.00159 & 0.0007 & 2.3249 & 0.02129 & $*$ \\
$\mathrm{I}($ Exotic *SpPt) & 0.00083 & 0.0007 & 1.1590 & 0.24811 & \\
$\mathrm{I}($ Pasturelands * SpPt) & 0.00127 & 0.0006 & 2.2893 & 0.02332 & $*$ \\
\hline
\end{tabular}

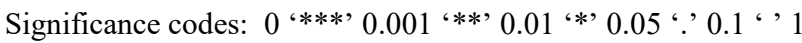

Residual standard error: 6.784 on 166 degrees of freedom

Multiple R-squared: 0.6407, Adjusted R-squared: 0.6256

F-statistic: 42.29 on 7 and $166 \mathrm{DF}, \mathrm{p}$-value: $<2.2 \mathrm{e}-16$

c) Multiple regression model for annual high flows (Y90m) considering alternative land cover and its interaction with annual precipitation (YP).

\begin{tabular}{lccrrc}
\hline & \multicolumn{3}{c}{ Std. } & & \\
& Estimate & Error & $\mathrm{t}$ value & $\mathrm{p}$ value & Significance \\
\hline (Intercept) & $-2.86 \mathrm{E}+02$ & $1.06 \mathrm{E}+02$ & -2.707 & 0.007451 & $* *$ \\
YP & $2.46 \mathrm{E}-01$ & $7.31 \mathrm{E}-02$ & 3.364 & 0.000941 & $* * *$
\end{tabular}




\begin{tabular}{lccccc} 
Native & $2.88 \mathrm{E}+00$ & $1.14 \mathrm{E}+00$ & 2.5281 & 0.01234 & $*$ \\
Exotic & $2.02 \mathrm{E}+00$ & $1.14 \mathrm{E}+00$ & 1.766 & 0.079105 &. \\
Pasturelands & $3.44 \mathrm{E}+00$ & $1.19 \mathrm{E}+00$ & 2.8874 & 0.004367 & $* *$ \\
$\mathrm{I}($ Native * YP) & $-2.07 \mathrm{E}-03$ & $7.54 \mathrm{E}-04$ & -2.7403 & 0.006764 & $* *$ \\
$\mathrm{I}($ Exotic *YP) & $-1.54 \mathrm{E}-03$ & $7.85 \mathrm{E}-04$ & -1.9637 & 0.051117 &. \\
$\mathrm{I}($ Pasturelands * YP) & $-2.45 \mathrm{E}-03$ & $8.50 \mathrm{E}-04$ & -2.8772 & 0.004503 & $* *$ \\
\hline
\end{tabular}

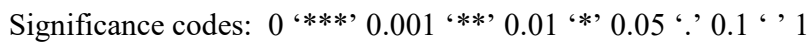

Residual standard error: 13.5 on 178 degrees of freedom

Multiple R-squared: 0.6837, Adjusted R-squared: 0.6713

F-statistic: 54.97 on 7 and $178 \mathrm{DF}$, p-value: $<2.2 \mathrm{e}-16$

d) Multiple regression model for low flows for spring ( $\mathrm{Sp} 10 \mathrm{~m})$ considering alternative land cover and its interaction with seasonal precipitation (spring + winter precipitation, $\mathrm{SpPt}$ ).

\begin{tabular}{lrrrrc}
\hline & Estimate & Std. Error & $\mathrm{t}$ value & $\mathrm{p}$ value & Significance \\
\hline (Intercept) & 65.57420 & 33.1084 & 1.9806 & 0.04929 & $*$ \\
SpPt (SpP+WP) & -0.06361 & 0.0458 & -1.3876 & 0.16712 & \\
Native & -0.99263 & 0.3915 & -2.5358 & 0.01214 & $*$ \\
Exotic & -0.65681 & 0.3678 & -1.7858 & 0.07596 & $*$ \\
Pasturelands & -0.80725 & 0.3327 & -2.4266 & 0.01631 & $*$ \\
$\mathrm{I}($ Native * SpPt) & 0.00109 & 0.0005 & 2.1315 & 0.03452 & $*$ \\
$\mathrm{I}($ Exotic *SpPt) & 0.00070 & 0.0005 & 1.3761 & 0.17065 & \\
$\mathrm{I}($ Pasturelands * SpPt) & 0.00108 & 0.0005 & 2.2733 & 0.02429 & $*$ \\
\hline
\end{tabular}

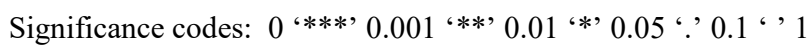

Residual standard error: 3.955 on 166 degrees of freedom

Multiple R-squared: 0.5759, Adjusted R-squared: 0.558

F-statistic: 32.2 on 7 and $166 \mathrm{DF}$, p-value: $<2.2 \mathrm{e}-16$ 